Bohdan Kopytko, Andriy Novosyadlo, Feller semigroup for diffusion process piesewise-constant generalized diffusion matrix and generalized drift vector, Scientific Research of the Institute of Mathematics and Computer Science, 2012, Volume 11, Issue 4, pages 75-84.

The website: http://www.amcm.pcz.pl/

Scientific Research of the Institute of Mathematics and Computer Science 4(11) 2012, 75-84

\title{
FELLER SEMIGROUP FOR DIFFUSION PROCESS PIESEWISE-CONSTANT GENERALIZED DIFFUSION MATRIX AND GENERALIZED DRIFT VECTOR
}

\author{
Bohdan Kopytko ${ }^{1}$, Andriy Novosyadlo ${ }^{2}$ \\ ${ }^{1}$ Institute of Mathematics, Czestochowa University of Technology, Czestochowa, Poland \\ ${ }^{2}$ Ivan Franko Lviv National University \\ bohdan.kopytko@im.pcz.pl,nandrew183@gmail.com
}

\begin{abstract}
We obtain an integral representation of the classical solution of the conjugation problem for the second order parabolic equation with derivatives with respect to tangent variables at the conjugation conditions. Using this solution, we construct the Feller semigroup to which there corresponds a diffusion process with a piecewise-constant generalized diffusion matrix and a generalized drift vector.
\end{abstract}

\section{Introduction}

In this paper, we consider the problem of construction of the Feller semigroup to which there corresponds a multidimensional continuous Markov process such that in the lower and upper halfspaces its parts coincide with given processes of Brownian motion and the behavior of the process, after its exit onto the common boundary of the given domains, is determined by two conjugation conditions given, that should be satisfied by the required semigroup. Brownian motion processes are given by their generators differential operators with zero transition vectors and distinct constants diffusion matrices. Note that the first of conjugation conditions is an expression of the Feller property of the required process, and the second condition corresponds to one of the versions of general conjugation condition of the Wentzel type (see [1,2]). In the considered case, the given condition is determined by a linear differential operator with constant coefficients, that contains normal derivatives and first and second order derivatives with respect to tangent variables. This means that, among the possible extensions of the process of Brownian motion at the points of the hyperplanethat separates the upper and lower halfspaces, there is only their partial reflection acting on the normal directions along with drift and diffusion along the boundary.

The formulated problem can also be called either a problem on gluing diffusion processes or a problem of construction of a mathematical model of diffusion phenomenon in the environment with diaphragm $[3,4]$. In the paper, we use analytical methods for its solution. With this approach, the given problem can be practically reduced to investigate the corresponding conjugation problem for a second order 
linear parabolic equation with discontinuous coefficients. We establish classical solvability of the latter problem by the method of the limiting integral equation using ordinary parabolic simple layer potential. Note also that the assumption that the coefficients of the equation and the Wentzel boundary operator are piecewiseconstant and constant respectively allow us to apply the Fourier-Laplace integral transformations to the solutions of the system of integral equations to which the initial problem is reduced.

Recall that a similar problem was already investigated earlier in [4]. However, a special fundamental solution was used therein which was constructed by the author, not an ordinary fundamental solution of a uniformly parabolic operator, as one of the kernels of the simple layer potential in construction of the integral representation of the regularized semigroup. We also distinguish the paper [5], where the problem of gluing of diffusion process is considered in martingale setting.

\section{Notations, the problem formulation}

The following notations are used in this paper: $\mathbb{R}=\mathbb{R}^{1}$ is a real line; $\mathbb{C}$ is a complex plane; $\mathbb{R}^{d}$ is a real $d$-dimensional Euclidean space of points $x=$ $=\left(x_{1}, \ldots, x_{d}\right), \quad|x|=\left(x_{1}^{2}+\cdots+x_{d}^{2}\right)^{1 / 2} ; \quad \mathcal{D}_{1}=\mathbb{R}_{-}^{d}=\left\{x \in \mathbb{R}^{d}: x_{d}<0\right\}, \quad \mathcal{D}_{2}=$ $=\mathbb{R}_{+}^{d}=\left\{x \in \mathbb{R}^{d}: x_{d}>0\right\}, \mathcal{S}=\mathbb{R}^{d-1} \equiv\left\{x \in \mathbb{R}^{d}: x_{d}=0\right\} ;$ points in $\mathbb{R}^{d-1}$ are noted as $x^{\prime}=\left(x_{1}, \ldots, x_{d-1}\right)$, such that $x=\left(x^{\prime}, x_{d}\right) ; \quad(x, y)=\sum_{i=1}^{d} x_{i} y_{i}$ for $\{x, y\} \subset \mathbb{R}^{d}$ and $\left(x^{\prime}, y^{\prime}\right)=\sum_{i=1}^{d-1} x_{i} y_{i}$ for $\left\{x^{\prime}, y^{\prime}\right\} \subset \mathbb{R}^{d-1} ; v\left(x^{\prime}\right)=\left(v_{i}\left(x^{\prime}\right)\right)_{i=1}^{d-1}$, $v_{i}\left(x^{\prime}\right)=0, i \in\{1, \ldots, d-1\}, v_{d}=1$ is a unit normal vector to $\mathcal{S}$ in the point $x^{\prime}$ directed inside the domain $\mathcal{D}_{2}$; points in $\mathbb{R}^{d+1}$ are denoted as $(t, x)=$ $=\left(t, x_{1}, \ldots, x_{d}\right)=\left(t, x^{\prime}, x_{d}\right)$, also $t$ is interpreted as time coordinate and $x_{1}, \ldots x_{d}$ are spatial values; $\mathbb{R}_{\infty}^{d+1} \equiv(0, \infty) \times \mathbb{R}^{d}, \mathbb{R}_{\infty}^{d} \equiv(0, \infty) \times \mathbb{R}^{d-1}, \Omega^{(l)} \equiv(0, \infty) \times$ $\mathcal{D}_{l}, l \in\{1,2\}$; for some fixed $T>0 R_{T}^{d+1} \equiv(0, T) \times \mathbb{R}^{d}, R_{T}^{d} \equiv(0, T) \times \mathbb{R}^{d-1}$, $\Omega_{T}^{(l)} \equiv(0, T) \times \mathcal{D}_{l}, l \in\{1,2\} ; \bar{Q}$ is a closure of a set $Q ; D_{t}^{1}=D_{t} \equiv \frac{\partial}{\partial t}, D_{i} \equiv \frac{\partial}{\partial x_{i}}$, $D_{i j} \equiv \frac{\partial^{2}}{\partial x_{i} \partial x_{j}},\{i, j\} \subset\{1, \ldots, d\}$, are operator of differentiation; $D_{t}^{r}$ and $D_{x}^{p}$ are the symbols of the partial derivative of the order $r$ with respect to $t$ and any partial of the order $p$ with respect to $x$ respectively, where $r$ and $p$ are nonnegative integers; $\nabla^{\prime}=\left(D_{1}, \ldots, D_{d-1}\right)$ is a spatial gradient; $\Delta_{x}^{\tilde{x}} f(\cdot, x)=f(\cdot, x)-f(\cdot, \tilde{x}), \Delta_{t}^{\tilde{t}} f(t, \cdot)=$ $=f(t, \cdot)-f(\tilde{t}, \cdot) ; \mathcal{C}(\Omega)(\mathcal{C}(\bar{\Omega}))$ is the set of all continuous functions on $\Omega(\bar{\Omega})$, where $\Omega$ is a subset of the region $\mathbb{R}_{\infty}^{d+1} ; \mathcal{C}^{1,2}(\Omega)\left(\mathcal{C}^{1,2}(\bar{\Omega})\right)$ is the set of all continuous functions on $\Omega(\bar{\Omega})$ that have continuous on $\Omega(\bar{\Omega})$ derivatives $D_{t}^{r}$ and $D_{x}^{p}$, $r=1, p \leq 2 ; \mathcal{C}_{b}\left(\mathbb{R}^{d}\right)$ is a space of bounded and continuous in $\mathbb{R}^{d}$ functions $\varphi$ with norm $\|\varphi\|=\sup _{\mathrm{x} \in \mathbb{R}^{\mathrm{d}}}|\varphi|$. By $\tilde{f}\left(t, \sigma^{\prime}\right)$ we will denote the Fourier transform by variable $x^{\prime}$ of the function $f\left(t, x^{\prime}\right)$, and by $\overline{\tilde{f}}\left(p, \sigma^{\prime}\right)$ we will denote a Laplace transform by variable $t$ of the function $\tilde{f}\left(t, \sigma^{\prime}\right)$ : 


$$
\overline{\tilde{f}}\left(p, \sigma^{\prime}\right) \equiv \int_{0}^{\infty} e^{-p t} d t \int_{\mathbb{R}^{d-1}} e^{i\left(x^{\prime}, \sigma^{\prime}\right)} f\left(t, x^{\prime}\right) d x^{\prime},
$$

$\sigma^{\prime} \in \mathbb{R}^{d-1}, p \in \mathbb{C}_{a}$, where $\mathbb{C}_{a} \equiv\{p \in \mathbb{C}: \operatorname{Re} p>a\}, a$ is some nonnegative constant that is defined by a function $f$. Everywhere below $C$ and $c$ are some positive constants that do not depend on $(t, x)$ and as a rule their values are not important to us. Other notations will be explained as soon as they have arisen.

Let us continue with the problem formulation. Assume that in the inner points of domains $\mathcal{D}_{1}=\mathbb{R}_{-}^{d}$ and $\mathcal{D}_{2}=\mathbb{R}_{+}^{d}, d \geq 2$, that are divided by a hyperplane $\mathcal{S}=\mathbb{R}^{d-1}$, are given generating differential operators of some Brownian motion processes $L_{1}$ and $L_{2}$ respectively:

$$
L_{l}=\frac{1}{2} \sum_{i, j=1}^{d} b_{i j}^{(l)}(x) \frac{\partial^{2}}{\partial x_{i} \partial x_{j}}, l=1,2,
$$

where $b_{i j}^{(l)}$ are real numbers and matrix $B_{l}=\left(b_{i j}^{(l)}\right)$ is symmetric positively defined. Assume also that numeric parameters $\beta_{i j}, \alpha_{i} \in \mathbb{R},\{i, j\} \subset\{1, \ldots, d-1\}$, and $q_{l}, l \in\{1,2\}$, with $q_{l} \geq 0$ and $q_{1}+q_{2} \neq 0$ are given, and matrix $\beta=\left(\beta_{i j}\right)$ is symmetrical and positively defined. They will be used to describe properties of the diaphragm that is situated on $\mathcal{S}$ and it affects a diffusing particle only when it reaches the diaphragm. We set up a problem to investigate the existence of a Feller semigroup $T_{t}, t \geq 0$, that generates a class of continuous Markov processes in $\mathbb{R}^{d}$ such that their parts in domains $\mathcal{D}_{l}$ coincide with Brownian motion processes that are controlled by the operator $L_{l}, l=1,2$.

In this paper, one such semigroup will be constructed by analytical methods under assumptions that the function $u(t, x)=T_{t} \varphi(x), \varphi \in \mathcal{C}_{b}\left(\mathbb{R}^{d}\right)$, is a solution of the next parabolic conjugation problem:

$$
\begin{gathered}
D_{t} u(t, x)-L_{l} u(t, x)=0, \quad(t, x) \in \Omega^{(l)}, l \in\{1,2\}, \\
u(0, x)=\varphi(x), \quad x \in \mathbb{R}^{d}, \\
u\left(t, x^{\prime}, 0-\right)=u\left(t, x^{\prime}, 0+\right), \quad\left(t, x^{\prime}\right) \in \overline{\mathbb{R}_{\infty}^{d}}, \\
L_{0} u\left(t, x^{\prime}, 0\right) \equiv \frac{1}{2} \sum_{i, j=1}^{d-1} \beta_{i j} D_{i j} u\left(t, x^{\prime}, 0\right)+\sum_{i=1}^{d-1} \alpha_{i} D_{i} u\left(t, x^{\prime}, 0\right)- \\
-q_{1} D_{d} u\left(t, x^{\prime}, 0-\right)+q_{2} D_{d} u\left(t, x^{\prime}, 0+\right)=0, \quad\left(t, x^{\prime}\right) \in \mathbb{R}_{\infty}^{d},
\end{gathered}
$$

where $u\left(t, x^{\prime}, 0-\right)\left(D_{d} u\left(t, x^{\prime}, 0-\right)\right)$ and $u\left(t, x^{\prime}, 0+\right)\left(D_{d} u\left(t, x^{\prime}, 0+\right)\right)$ are boundary values of the function $u\left(t, x^{\prime}, 0\right)\left(D_{d} u\left(t, x^{\prime}, 0\right)\right)$ as $x$ approaches to $\left(x^{\prime}, 0\right) \in$ $\mathbb{R}^{d-1}$ from inside $\mathcal{D}_{1}$ and $\mathcal{D}_{2}$ respectively. 
Note that if the semigroup satisfies equation (2) then the sought process coincides with given Brownian motion processes in $\mathcal{D}_{1}$ and $\mathcal{D}_{2}$; also the equation (3) corresponds to the fact that $T_{0} \equiv I$, where $I$ is an identity operator. Besides that, the formulation of the problem (2) - (5) requires that the function $u(t, x)$ and its derivatives $D_{i} u(t, x), i \in\{1, \ldots, d\}$, change continuously as it moves through $\mathcal{S}$. Probabilistic interpretation of conditions (4) and (5) and proper comments were stated in the introduction.

So, we are interested in classical solution of the problem (2)-(5) that is bounded by a spatial variable and the function $u(t, x)$ belongs to

$$
\mathcal{C}^{1,2}\left(\Omega^{(l)}\right) \cap \mathcal{C}\left(\overline{\Omega_{\infty}^{d+1}}\right),
$$

and its derivatives $D_{i} u(t, x), D_{i j} u(t, x),\{i, j\} \subset\{1, \ldots, d-1\}$, exist and are continuous in all points of the domain $(t, x) \in \mathbb{R}_{\infty}^{d+1}$.

\section{Solution of the parabolic conjugation problemand construction of generalized diffusion process}

We will use a method of boundary integral equations to prove a classical solvability of the problem (2)-(5).

Theorem 1. Let the coefficients of the operators $L_{l}, l \in\{1,2\}$ from the problem (2)-(5) are real constants that construct a positively defined symmetric matrix $B_{l}$, and numeric parameters $\beta_{i j}, \alpha_{i},\{i, j\} \subset\{1, \ldots, d-1\}$, and $q_{l}, l \in\{1,2\}$, satisfy the next conditions: $\beta_{i j} \in \mathbb{R}, \alpha_{i} \in \mathbb{R}, q_{l} \geq 0, q_{1}+q_{2} \neq 0$ and the matrix $\beta=\left(\beta_{i j}\right)$ is symmetric and positively defined. Then the problem (2)-(5) has a unique classical solution for every function $\varphi \in \mathcal{C}_{b}\left(\mathbb{R}^{d}\right)$ and next estimation holds:

$$
|u(t, x)| \leq C\|\varphi\|, \quad(t, x) \in \overline{\mathbb{R}_{T}^{d+1}} .
$$

The proof of the theorem is in receiving an explicit form of the solution as a sum of Poisson potential and simple layer potential, then a respective estimates are proved. Let $g_{l}(t, x, y), l \in\{1,2\}, t>0, x \in \mathbb{R}^{d}, y \in \mathbb{R}^{d}$ is a fundamental solution (f.s.) of the equation (2):

$$
\begin{aligned}
& g_{l}(t, x, y)=g_{l}(t, x-y)=g_{l}\left(t, x^{\prime}-y^{\prime}, x_{d}-y_{d}\right)= \\
& \quad=(2 \pi t)^{-\frac{d}{2}}\left(\operatorname{det} B_{l}\right)^{-\frac{1}{2}} \exp \left(-\frac{1}{2 t}\left(B_{l}^{-1}(x-y), x-y\right)\right) .
\end{aligned}
$$

Consider the Poisson potential and simple layer potential

$$
u_{0 l}(t, x)=\int_{\mathbb{R}^{d}} g_{l}(t, x, y) \varphi(y) d y, \quad(t, x) \in \mathbb{R}_{\infty}^{d+1}, \quad l \in\{1,2\},
$$




$$
u_{1 l}(t, x)=\int_{0}^{t} d \tau \int_{\mathbb{R}^{d-1}} g_{l}\left(t-\tau, x, y^{\prime}\right) V_{l}\left(\tau, y^{\prime}, \varphi\right) d y^{\prime},(t, x) \in \mathbb{R}_{\infty}^{d+1}, l \in\{1,2\}
$$

Here $\varphi$ is the function from (3), and density functions $V_{l}, l \in\{1,2\}$, are to be defined. Notice that dependence of densities $V_{l}, l \in\{1,2\}$ on initial function $\varphi$ from (3) will be defined in the problem by conjugation conditions (4), (5).

Let us note some properties of the potentials $u_{0 l}, u_{1 l}, l \in\{1,2\}$, that follows directly from the properties of f.s. $g_{0 l}$ (see $[6, \mathrm{Ch}$. IV] and $[7,8]$ ). In particular, if $\varphi \in \mathcal{C}_{b}\left(\mathbb{R}^{d}\right)$, then the functions $u_{0 l}(t, x), l \in\{1,2\}$, satisfy equation (2) in domains $(t, x) \in \Omega^{(l)}$, initial condition (3), and in every domain of next form $(t, x)$ $\in(0, T] \times \mathbb{R}^{d}$ next inequality holds

$$
\left|D_{t}^{r} D_{x}^{p} u_{0 l}(t, x)\right| \leq C\|\varphi\| t^{-\frac{2 r+p}{2}},
$$

where $r$ and $p$ are nonnegative integers.

Further, let us assume a priori that functions $V_{l}\left(t, x^{\prime}, \varphi\right) \equiv V_{l}\left(t, x^{\prime}\right), l \in\{1,2\}$, arecontinuous in the domain $\left(t, x^{\prime}\right) \in \mathbb{R}_{\infty}^{d}$, and when $\left(t, x^{\prime}\right) \in(0, T] \times \mathbb{R}^{d-1}$ next inequalities holds

$$
\left|V_{l}\left(t, x^{\prime}\right)\right| \leq C\|\varphi\| t^{-\frac{1}{2}} .
$$

Then the functions $u_{1 l}(t, x), l \in\{1,2\}$, satisfy the equation (2) in domains $(t, x) \in$ $\Omega^{(l)}$, initial condition $u_{1 l}(0, x)=0, x \in \mathbb{R}^{d}$, and next inequality holds

$$
\left|u_{1 l}(t, x)\right| \leq C\|\varphi\|, \quad(t, x) \in(0, T] \times \mathbb{R}^{d} .
$$

We will also use formulas of jump of conormal derivatives of simple layer potential on the boundary $\mathcal{S}=\mathbb{R}^{d-1}$. For this, for $x^{\prime} \in \mathbb{R}^{d-1}$, we define vectors $N_{l}=B_{l} \cdot v, l \in\{1,2\}$, that are called conormals. Due to the assumption of the Theorem $1\left(B_{l} v, v\right)>0, l \in\{1,2\}$, so that both conormals have directions inside the domain $\mathcal{D}_{2}$. A derivative of some function $v(x), x \in \mathbb{R}^{d}$, in the direction of every conormal $N_{l}, l \in\{1,2\}$, is defined by a formula

$$
\frac{\partial v(x)}{\partial N_{l}}=\sum_{i=1}^{d} b_{i d}^{(l)} D_{i} v(x) .
$$

In the sought case for conormal derivatives of simple layer potentials $u_{1 l}(t, x)$, $l \in\{1,2\}$, for $t>0$ and $x=\left(x^{\prime}, 0\right) \in \mathbb{R}^{d-1}$ we will obtain

$$
\frac{\partial u_{1 l}\left(t, x^{\prime}, 0 \mp\right)}{\partial N_{l}}= \pm V_{l}\left(t, x^{\prime}\right), \quad l \in\{1,2\} .
$$

As potentials $u_{0 l}$ and $u_{1 l}, l \in\{1,2\}$, satisfy the mentioned conditions then we can try to find a solution of the problem (2)-(5) in next form 


$$
u(t, x)=u_{l}(t, x), \quad(t, x) \in \Omega^{(l)}, \quad l \in\{1,2\},
$$

where

$$
u_{l}(t, x)=u_{0 l}(t, x)+u_{1 l}(t, x) .
$$

Now we have to find unknown functions $V_{1}$ and $V_{2}$ such that conjugation conditions (4) and (5) are satisfied for $u(t, x)$. Substituting expressions for $u_{l}(t, x)$, $l \in\{1,2\}$ in these conditions after some transformations we will obtain

$$
\begin{gathered}
\sum_{l=1}^{2}(-1)^{l-1} \int_{0}^{t} d \tau \int_{\mathbb{R}^{d-1}} g_{l}\left(t-\tau, x^{\prime}-y^{\prime}, 0\right) V_{l}\left(\tau, y^{\prime}\right) d y^{\prime}= \\
=u_{02}\left(t, x^{\prime}, 0\right)-u_{01}\left(t, x^{\prime}, 0\right), \\
\sum_{l=1}^{2} \gamma_{l}\left[\left(\frac{1}{2} \sum_{i, j=1}^{d-1} \beta_{i j}^{(0)} D_{i j}+\sum_{i=1}^{d-1} \alpha_{i}^{(0)} D_{i}\right) u_{0 l}\left(t, x^{\prime}, 0\right)+\right. \\
+(-1)^{l} \frac{\partial u_{0 l}\left(t, x^{\prime}, 0\right)}{\partial N_{l}}-\frac{V_{l}\left(t, x^{\prime}\right)}{b_{d d}^{(l)}}+\left(\frac{1}{2} \sum_{i, j=1}^{d-1} \beta_{i j}^{(0)} D_{i j}+\right. \\
\left.\left.+\sum_{i=1}^{d-1} \alpha_{i}^{(0)} D_{i}\right) \int_{0}^{t} d \tau \int_{\mathbb{R}^{d-1}} g_{l}\left(t-\tau, x^{\prime}-y^{\prime}, 0\right) V_{l}\left(\tau, y^{\prime}\right) d y^{\prime}\right]=0,
\end{gathered}
$$

where

$$
\begin{gathered}
\alpha_{i}^{(0)}=\frac{\alpha_{i}}{q_{2}+q_{1}}+\sum_{l=1}^{2}(-1)^{l-1} \frac{\gamma_{l} b_{i d}^{(l)}}{b_{d d}^{(l)}}, i \in\{1, \ldots, d-1\}, \\
\beta_{i j}^{(0)}=\frac{\beta_{i j}}{q_{2}+q_{1}},\{i, j\} \subset\{1, \ldots, d-1\}, \gamma_{1}=\frac{1-q}{2}, \gamma_{2}=\frac{1+q}{2}, \quad q=\frac{q_{2}-q_{1}}{q_{2}+q_{1}} .
\end{gathered}
$$

So from conjugation conditions (4), (5) we have obtained a system of equations (16), (17) with respect to unknown functions $V_{l}, l \in\{1,2\}$. As one can see, the first equation is an integral Volterra equation of the first kind, and the second one is an integral-differential Volterra equation of the second kind. We will show that the system of equations (16), (17) can be solved using the integral Fourier transformwith respect to the variable $x^{\prime}$ and the integral Laplace transform with respect to variable $t$. We have agreed to denote Fourier-Laplace transforms of the function $f\left(t, x^{\prime}\right)$ by $\overline{\tilde{f}}\left(p, \sigma^{\prime}\right)$ (see Ch. 2). Obviously we will consider that this transformation exists for every function from equations (16) and (17). After application of the Fourier-Laplace transform to every equation in the system (16) and (17) it transforms into algebraic system of equations with respect to the images $\overline{\widetilde{V}}_{l}\left(p, \sigma^{\prime}\right)$, $l \in\{1,2\}$, and we will obtain 


$$
\begin{array}{r}
\overline{\widetilde{V}}_{l}\left(p, \sigma^{\prime}\right)=2 b_{d d}^{(l)}\left(p+a_{l}\right)\left[-\overline{\tilde{g}}_{l}\left(p, \sigma^{\prime}, 0\right) \overline{\tilde{u}}_{0 l}\left(p, \sigma^{\prime}, 0\right)\right. \\
\left.+\tilde{\tilde{G}}_{l}\left(p, \sigma^{\prime}\right) \tilde{\tilde{\psi}}\left(p, \sigma^{\prime}\right)\right], \quad l \in\{1,2\},
\end{array}
$$

where

$$
\begin{gathered}
\overline{\tilde{\psi}}\left(p, \sigma^{\prime}\right)=\left.\frac{2 \gamma_{2}}{b_{d d}^{(2)}} \int_{0}^{\infty} \frac{\partial \overline{\tilde{g}}_{2}\left(p, \sigma^{\prime}, x_{d}-y_{d}\right)}{\partial N_{2}}\right|_{x_{d}=0} \cdot \tilde{\varphi}\left(\sigma^{\prime}, y_{d}\right) d y_{d}- \\
-\left.\frac{2 \gamma_{1}}{b_{d d}^{(1)}} \int_{-\infty}^{0} \frac{\partial \overline{\tilde{g}}_{1}\left(p, \sigma^{\prime}, x_{d}-y_{d}\right)}{\partial N_{1}}\right|_{x_{d}=0} \cdot \tilde{\varphi}\left(\sigma^{\prime}, y_{d}\right) d y_{d}, \\
\overline{\tilde{G}}_{l}\left(p, \sigma^{\prime}\right)=\left(2 b_{d d}^{(l)}\left(p+a_{l}\right)\right)^{-\frac{1}{2}}\left[\gamma_{1} \sqrt{\frac{2}{b_{d d}^{(1)}}\left(p+a_{1}\right)}+\gamma_{2} \sqrt{\frac{2}{b_{d d}^{(2)}}\left(p+a_{2}\right)}+K\right]^{-1}, \\
a_{l}=\frac{1}{2}\left(H_{l} \sigma^{\prime}, \sigma^{\prime}\right), \quad H_{l}=\left(h_{i j}^{(l)}\right)_{i, j=1}^{d-1}, \quad h_{i j}^{(l)}=b_{i j}^{(l)}-\frac{b_{i d}^{(l)} b_{j d}^{(l)}}{b_{d d}^{(l)}}, \\
K=\frac{1}{2}\left(\beta_{0} \sigma^{\prime}, \sigma^{\prime}\right)+i\left(\alpha_{0}^{\prime}, \sigma^{\prime}\right), \quad \beta_{0}=\left(\beta_{i j}^{(0)}\right)_{i, j=1}^{d-1}, \quad \alpha_{0}^{\prime}=\left(\alpha_{i}^{(0)}\right)_{i=1}^{d-1} .
\end{gathered}
$$

Notice that for obtaining and solving the system with unknown $\overline{\widetilde{V}}_{l}\left(p, \sigma^{\prime}\right), l \in\{1,2\}$ we have used known properties of Fourier and Laplace transforms [9] along with the next relation for image of f.s. which was established by us $g_{l}(t, x)=$ $=g_{l}\left(t, x^{\prime}, x_{d}\right), l \in\{1,2\}$ :

$$
\overline{\widetilde{g}}_{l}\left(p, \sigma^{\prime}, x_{d}\right)=\left(2 b_{d d}^{(l)}\left(p+a_{l}\right)\right)^{-\frac{1}{2}} \exp \left\{i x_{d} \sum_{j=1}^{d-1} \frac{b_{j d}^{(l)} \sigma_{j}}{b_{d d}^{(l)}}-\left|x_{d}\right|\left(\frac{2}{b_{d d}^{(l)}}\left(p+a_{l}\right)\right)^{\frac{1}{2}}\right\} .
$$

From equalities (18) and well-known formula for the Fourier-Laplace transform of convolution of functions follows that the solution of the system (16)-(17) can be written in next form

$$
\begin{gathered}
V_{l}\left(t, x^{\prime}\right)=2 b_{d d}^{(l)} \mu_{l}\left(t, x^{\prime}\right) \int_{0}^{t} d \tau \int_{\mathbb{R}^{d-1}}\left[-g_{l}\left(t-\tau, x^{\prime}-y^{\prime}, 0\right) \times u_{0 l}\left(\tau, y^{\prime}, 0\right)\right. \\
\left.+G_{l}\left(t-\tau, x^{\prime}-y^{\prime}\right) \psi\left(\tau, y^{\prime}\right)\right] d y^{\prime}, \quad l \in\{1,2\}
\end{gathered}
$$

where $\mu_{l}$ are uniformly parabolic operators,

$$
\begin{gathered}
\mu_{l}=D_{t}-\frac{1}{2} \sum_{i, j=1}^{d-1} h_{i j}^{(l)} D_{i j}, \\
\psi\left(\tau, y^{\prime}\right)=\sum_{l=1}^{2}(-1)^{l} \frac{2 \gamma_{l}}{b_{d d}^{(l)}} \int_{\mathcal{D}_{l}} \frac{\partial g_{l}\left(\tau, y^{\prime}, z\right)}{\partial N_{l}} \varphi(z) d z,
\end{gathered}
$$


and $G_{l}\left(t, x^{\prime}\right)$ are originals of the functions $\overline{\widetilde{G}}_{l}\left(p, \sigma^{\prime}\right)$. We will use next equality to find them

$$
\begin{aligned}
\overline{\widetilde{G}}_{l}\left(p, \sigma^{\prime}\right)=\int_{0}^{\infty} & \exp \{-K u\}\left(2 b_{d d}^{(l)}\left(p+a_{l}\right)\right)^{-\frac{1}{2}} \exp \left\{-u\left[\gamma_{1} \sqrt{\frac{2}{b_{d d}^{(1)}}\left(p+a_{1}\right)}\right.\right. \\
& \left.\left.+\gamma_{2} \sqrt{\frac{2}{b_{d d}^{(2)}}\left(p+a_{2}\right)}\right]\right\} .
\end{aligned}
$$

Hence we have

$$
G_{l}\left(t, x^{\prime}\right)=\int_{0}^{\infty} d u \int_{\mathbb{R}^{d-1}} G_{0 l}\left(t, x^{\prime}-y^{\prime}, u\right) g_{0}\left(u, y^{\prime}\right) d y^{\prime}, \quad l \in\{1,2\},
$$

where

$$
\begin{gathered}
G_{0 l}\left(t, x^{\prime}, u\right)=\Gamma_{l}\left(t, x^{\prime}, u\right)=\exp \left\{-\frac{\left(\gamma_{l} u\right)^{2}}{2 b_{d d}^{(l)} t}\right\} g_{0 l}\left(t, x^{\prime}, 0\right), \\
\text { if } \gamma_{3-l}=0, \\
G_{0 l}\left(t, x^{\prime}, u\right)=\frac{-b_{d d}^{(3-l)}}{\gamma_{3-l}} \int_{0}^{t} d \tau \int_{\mathbb{R}^{d-1}} \Gamma_{l}\left(t-\tau, x^{\prime}-y^{\prime}, u\right) D_{u} \Gamma_{3-l}\left(\tau, y^{\prime}, u\right) d y^{\prime}, \text { if } \\
\gamma_{3-l} \neq 0,
\end{gathered}
$$

$g_{0}\left(u, y^{\prime}\right)$ is a f.s. of uniformly parabolic operator

$$
D_{u}-\frac{1}{2} \sum_{i, j=1}^{d-1} \beta_{i j}^{(0)} D_{i j}-\sum_{i=1}^{d-1} \alpha_{i}^{(0)} D_{i}
$$

So we have found the solution of the system of equations (16), (17). Let us establish the estimation (10). For this purpose we will represent functions $V_{l}$, $l \in\{1,2\}$, in a form of a sum of two terms $V_{l 1}$ and $V_{l 2}$, where

$$
V_{l 1}\left(t, x^{\prime}\right)=-2 b_{d d}^{(l)} \mu_{l}\left(t, x^{\prime}\right) \int_{0}^{t} d \tau \int_{\mathbb{R}^{d-1}} \mathrm{~g}_{l}\left(t-\tau, x^{\prime}-y^{\prime}, 0\right) u_{0 l}\left(\tau, y^{\prime}, 0\right) d y^{\prime},
$$

and $V_{l 2}\left(t, x^{\prime}\right)$ is represented by the same formula only we have an integral from the product of the kernel $G_{l}$ and density $\psi$. Let us investigate functions $V_{l 1}$ more precisely. If we will consider the equality

$$
g_{l}\left(t-\tau, x^{\prime}-y^{\prime}, 0\right)=\left(2 \pi b_{d d}^{(l)}(t-\tau)\right)^{-1 / 2} h_{l}\left(t-\tau, x^{\prime}-y^{\prime}\right),
$$

where $h_{l}$ denotes a f.s. of the operator $\mu_{l}$, then it is easy to obtain next form for $V_{l 1}$ 


$$
\begin{gathered}
\left(\frac{\pi}{2 b_{d d}^{(l)}}\right)^{\frac{1}{2}} V_{l 1}\left(t, x^{\prime}\right)=\int_{0}^{t / 2} \frac{1}{2}(t-\tau)^{-\frac{3}{2}} d \tau \times \\
\times \int_{\mathbb{R}^{d-1}} h_{l}\left(t-\tau, x^{\prime}-y^{\prime}\right) u_{0 l}\left(\tau, y^{\prime}, 0\right) d y^{\prime}+\int_{\frac{t}{2}}^{t} \frac{1}{2}(t-\tau)^{-\frac{3}{2}} d \tau \times \\
\times \int_{\mathbb{R}^{d-1}} h_{l}\left(t-\tau, x^{\prime}-y^{\prime}\right)\left(u_{0 l}\left(\tau, y^{\prime}, 0\right)-u_{0 l}\left(\tau, x^{\prime}, 0\right)-\right. \\
\left.-\left(\nabla_{x^{\prime}}^{\prime} u_{0 l}\left(\tau, x^{\prime}, 0\right), y^{\prime}-x^{\prime}\right)\right) d y^{\prime}+\int_{\frac{t}{2}}^{t} \frac{1}{2}(t-\tau)^{-\frac{3}{2}} \times \\
\times\left(u_{0 l}\left(\tau, x^{\prime}, 0\right)-u_{0 l}\left(t, x^{\prime}, 0\right)\right) d \tau-\left(\frac{t}{2}\right)^{-\frac{1}{2}} u_{0 l}\left(t, x^{\prime}, 0\right) .
\end{gathered}
$$

After we estimate every term in the right part of (21) using inequalities (9) and known estimations for f.s. $h_{l}, l \in\{1,2\}$, we assure that inequality (10) holds for $V_{l 1}\left(t, x^{\prime}\right)$ with $\left(t, x^{\prime}\right) \in(0, T] \times \mathbb{R}^{d-1}$. Similarly one can investigate functions $V_{l 2}\left(t, x^{\prime}\right), l \in\{1,2\}$, and as consequence obtain estimation (10) for them. This means that our a priori assumptions on densities $V_{l}, l \in\{1,2\}$, that are included in simple layer potential $u_{1 l}$ from (8), holds true. From it and from inequalities (9) and (10) follows estimation (6) for the constructed solution of the problem (2)-(5). Theorem 1 is proved.

From Theorem 1 follows that an operator semigroup $\left(T_{t}\right)_{t \geq 0}$, on functions $\varphi \in \mathcal{C}_{b}\left(\mathbb{R}^{d}\right)$ can be defined by a relation $T_{t} \varphi(x)=u(t, x, \varphi)$, where the function $u$ is defined by formulas (14), (15), (19). Using these formulas and acting like in $[3,4,10]$, we prove that the semigroup, constructed this way, generates some homogeneous non-breaking Feller process on $\mathbb{R}^{d}$. Further, an additional investigation of the semigroup shows that trajectories of the constructed process can be considered to be continuous and its transition probability $P(t, x, d y)$ satisfies the next relations:

$$
\begin{gathered}
\lim _{t \downarrow 0} \int_{\mathbb{R}^{d}} \varphi(x)\left(\frac{1}{t} \int_{\mathbb{R}^{d}}(y-x, \Theta) P(t, x, d y)\right) d x= \\
=c(\bar{\alpha}, \Theta) \int_{\mathbb{R}^{d-1}} \varphi\left(x^{\prime}, 0\right) d x^{\prime}, \\
\lim _{t \downarrow 0} \int_{\mathbb{R}^{d}} \varphi(x)\left(\frac{1}{t} \int_{\mathbb{R}^{d}}(y-x, \Theta)^{2} P(t, x, d y)\right) d x= \\
=\int_{\mathbb{R}^{d}} \varphi(x)(B(x) \Theta, \Theta) d x \\
+\mathrm{c}(\bar{\beta} \Theta, \Theta) \int_{\mathbb{R}^{d-1}} \varphi\left(x^{\prime}, 0\right) d x^{\prime},
\end{gathered}
$$


where $\Theta \in \mathbb{R}^{d}, \varphi$ is an arbitrary continuous compact function on $\mathbb{R}^{d}$,

$$
\begin{gathered}
c=\frac{1}{2} \frac{\left(\sqrt{b_{d d}^{(1)}}+\sqrt{b_{d d}^{(2)}}\right) \sqrt{b_{d d}^{(1)} b_{d d}^{(2)}}}{q_{1} \sqrt{b_{d d}^{(2)}}+q_{2} \sqrt{b_{d d}^{(1)}}}, \\
\bar{\alpha}=\left(\bar{\alpha}_{i}\right)_{i=1}^{d}, \bar{\alpha}_{i}=\alpha_{i}, \quad i \in\{1, \ldots, d-1\}, \quad \bar{\alpha}_{d}=q_{1}+q_{2}, \\
B(x)=B_{l}, \quad x \in \mathcal{D}_{l}, \quad l \in\{1,2\}, \\
\bar{\beta}=\left(\bar{\beta}_{i j}\right)_{i, j=1}^{d}, \bar{\beta}_{i j}=\left\{\begin{array}{c}
\beta_{i j}, \quad \text { if }\{i, j\} \subset\{1, \ldots, d-1\}, \\
0, \quad \text { if } i=d \text { or } j=d .
\end{array}\right.
\end{gathered}
$$

Equalities (22) means that for the constructed process its local diffusion characteristics exist only as generalized functions, this means that this process is a generalized diffusion process in the sense of Portenko [10].

So we have proved next theorem.

Theorem 2. Let the coefficients of differential operators $L_{l}, l \in\{1,2\}$, from (1) and $L_{0}$ from (5) satisfy the conditions of Theorem 1. Then an operator semigroup, constructed by the solution of the conjugation problem (2)-(5), generates a homogeneous continuous Feller process on $\mathbb{R}^{d}$ with transition probability that satisfies relation (22).

\section{References}

[1] Wentzel A.D., On Boundary Conditions for Multidimensional Diffusion Processes, Theor. Prob. And Appl. 1959, 2, 5, 172-185 (in Russian).

[2] Dynkin E.B., Markov Processes, Academie Press, Springer, New York 1965.

[3] Portenko M.I., Diffusion Processes in Media with Membranes, Institute of Mathematics, of Ukrainian National Acad. of Sci., Kyiv 1995 (in Ukrainian).

[4] Kopytko B.I., Diffusion Processes with generalized drift vector and diffusion matrix, Proceedings of the $6^{\text {th }}$ USSR-Japan Symposium Probability Theory and Mathematical Statistics (Kiev, USSR, August 5-10, 1991), Singapore - New Jersey - London - Hong Kong 1992, 169-175.

[5] Anulova S.V., Diffusion Processes: Discontinuous Coefficients, Degenerate Diffusion, Randomized Drift, DAN USSR 1981, 260, 5, 1036-1040 (in Russian).

[6] Ladyzhenskaya O.A., Solonnikov V.A., Ural'tseva N.N., Linear and Quasilinear Equations of Parabolic Type, Nauka, Moscow 1967 (in Russian).

[7] Pogorzelski W., Étude de la solution fondamental de l'equationparabolique, Richerche di Mat. 1956, 5, 25-27.

[8] Pogorzelski W., Study of Integrals of Parabolic Equation and Boundary Problems in an Unbounded Domain, Math. Coll. 1959, 47(89), 4, 397-430 (in Russian).

[9] Bateman H., Erdélyi A., Tables of integral transforms, New York, Toronto, London, McGrawHill Book Company, Inc., 1954.

[10] Portenko N.I. , Generalized Diffusion Processes, Kyiv 1982 (English transl., Amer. Math. Soc., Providence, RI, 1990). 